\title{
The multiperiodicity of the $\gamma$ Doradus stars HD 224945 and HD 224638 as detected from a multisite campaign ${ }^{\star}$
}

\author{
E. Poretti ${ }^{1}$, C. Koen ${ }^{2}$, M. Bossi ${ }^{1}$, E. Rodríguez ${ }^{3}$, S. Martín ${ }^{3,1}$, K. Krisciunas ${ }^{4}$, M. C. Akan ${ }^{5}$, R. Crowe ${ }^{6}$, \\ M. Wilcox ${ }^{6}$, C. Ibanoglu ${ }^{5}$, and S. Evren ${ }^{5}$ \\ 1 Osservatorio Astronomico di Brera, Via Bianchi 46, 23807 Merate, Italy \\ 2 South African Astronomical Observatory, PO Box 9, Observatory 7935, Cape Town, South Africa \\ 3 Instituto de Astrofisica de Andalucia, C.S.I.C., Apdo. 3004, 18080 Granada, Spain \\ 4 Cerro Tololo Inter-American Observatory, Casilla 603, La Serena, Chile \\ ${ }^{5}$ Ege University, Science Faculty, Dept. of Astronomy and Space Sciences, Bornova 35100 Izmir, Turkey \\ 6 Department of Physics and Astronomy, University of Hawaii - Hilo, 200 West Kawili Street, Hilo, Hawaii, \\ 96720-4091, USA
}

Received 11 September 2001 / Accepted 9 January 2002

\begin{abstract}
We discuss new photometric data collected on the $\gamma$ Dor variables HD 224945 and HD 224638 . Multiperiodicity was detected in both stars, thanks to the clear spectral window of a multisite campaign that involved five observatories. HD 224945 shows the shortest period among the $\gamma$ Dor stars, i.e., 0.3330 d. The pulsation behaviour is very different: HD 224945 displays a set of frequencies spread over an interval much larger than that of HD 224638. We clearly found evidence for amplitude variations in the excited modes by comparing data from different years. HD 224945 and HD 224638 are among the best examples of $\gamma$ Dor stars that show multimode pulsations, which make them very interesting from an asteroseismological point of view.
\end{abstract}

Key words. methods: data analysis - stars: oscillations - stars: variables: general - techniques: photometric

\section{Introduction}

The variability of HD $224638 \equiv \mathrm{BT}$ Psc $(V=7.5, \mathrm{~F} 1 \mathrm{~V})$ and HD $224945 \equiv \mathrm{BU}$ Psc $(V=6.93$, F0 V) was announced by Mantegazza \& Poretti (1991), as a by-product of the monitoring of the $\delta$ Sct star HD $224639 \equiv$ BH Psc. Both stars had been used as comparison stars in the first observing run devoted to BH Psc. They increased the number of known $F$-type stars located close to the lowtemperature edge of the Cepheid instability strip which exhibit small amplitude variability on time scales of several hours, usually longer than the length of a night of observation and therefore easily detectable only when used as comparison stars for short-period variables. At that time, the debate on the nature of these variations was divided between spot activity (observed periods as rotational periods) and pulsation (nonradial $g$-modes). Mantegazza et al. (1994, hereinafter Paper I) tried to explain the complicated light behaviour of HD 224639 and HD 224945 in the simplest way possible, by means of periodicities shaped as double- or triple-wave curves. Balona et al. (1994) reported on the multiperiodicity of $\gamma$ Dor, giving decisive

Send offprint requests to: E. Poretti,

e-mail: poretti@merate.mi.astro.it

* Based on observations partially collected at ESO-La Silla (Proposals 54.E-018 and 56.E-0308). evidence in favour of pulsation. Also, as a result of combined photometry and line profile variations for 9 Aurigae (Krisciunas et al. 1995) and $\gamma$ Dor (Balona et al. 1996), plus the preliminary results on HD 224945 (Poretti et al. 1996), the hypothesis of variability caused by $g$-modes achieved a wide consensus. The properties of this new class of variable stars were delineated step-by-step through observational efforts and have been summarized by Kaye et al. (1999), while the problem of the driving mechanism and the excitation of $g$-modes constitutes the target of continuing theoretical investigations. As a result, $\gamma$ Dor variables are now considered intermediate between $A-F$ pulsators and solar-type stars, finding a special place in the main programmes of asteroseismologic missions such as COROT and MONS.

On the basis of this progress, it is worth investigating in more detail the pulsational behaviour of HD 224639 and HD 224945. Here we present the results of a multisite campaign carried out in October 1995.

\section{Observations}

HD 224945 and HD 224638 are located very close to the celestial equator and therefore can be monitored from both the northern and southern hemispheres. As comparison stars we used the same two stars as in the 1991 campaign 
(HD 225086 and HD 200), since they proved to be stable within a few mmag. Five telescopes were used for this multisite campaign:

1. The European Southern Observatory 50-cm telescope located in La Silla (Chile), equipped with a photoncounting photometer (EMI 9789 QB photomultiplier) and $B$ and $V$ filters. The observer was E. Poretti;

2. The $48-\mathrm{cm}$ telescope, located at Ege University Observatory, was equipped with a solid-state photometer and $B$ and $V$ filters. The observer team was led by M. C. Akan;

3. The $50-\mathrm{cm}$ telescope located in Sutherland, South African Astronomical Observatory, was equipped with a photon-counting photometer and $B$ and $V$ filters. The observer was C. Koen;

4. The 61-cm telescope located on the Mauna Kea (Hawaii) was equipped with a photon-counting photometer and standard $B$ and $V$ filters. The observers were K. Krisciunas, R. Crowe and M. Wilcox;

5. The 90-cm telescope located at Sierra Nevada Observatory (Spain). The observers were E. Rodríguez and S. Martín. Photometry was performed in the uvby system, but only $v$ and $y$ measurements are discussed here, as the more compatible with the $B$ and $V$ ones, respectively.

Earlier observations were performed in 1994 at European Southern Observatory, using the ESO $50-\mathrm{cm}$ telescope equipped with the same instrumentation used in 1995. On that occasion the observer was M. Bossi; the results obtained on BH Psc are reported by Mantegazza et al. (1996). The measurements were distributed over 13 nights, with a total useful observing time of 64 hours and a baseline of $14.9 \mathrm{~d}$.

\section{Data reduction and analysis}

The original measurements were provisionally reduced by the observers themselves to check the data quality and for comparison purposes. The original raw data were then re-reduced by means of the same algorithm; this homogenous procedure allowed us to avoid the introduction of local drifts caused by routines using different interpolation formulae and/or different methods of applying extinction corrections. Since the weather was not particularly favourable during the campaign, the rejection of some parts of nights characterized by unfavourable weather conditions was decided on the basis of a uniform criterion. We also decided to use the code which allows us to calculate instantaneous values of the extinction coefficients (Poretti \& Zerbi 1993) since in some cases changes were expected to occur.

Table 1 lists some details regarding observing runs at each site. As can be noted, the mean values of the magnitude differences between the two comparison stars show the presence of systematic shifts (up to $0.03 \mathrm{mag}$ ). This is not surprising, taking into account that different photomultipliers and filters were used. The systematic shifts

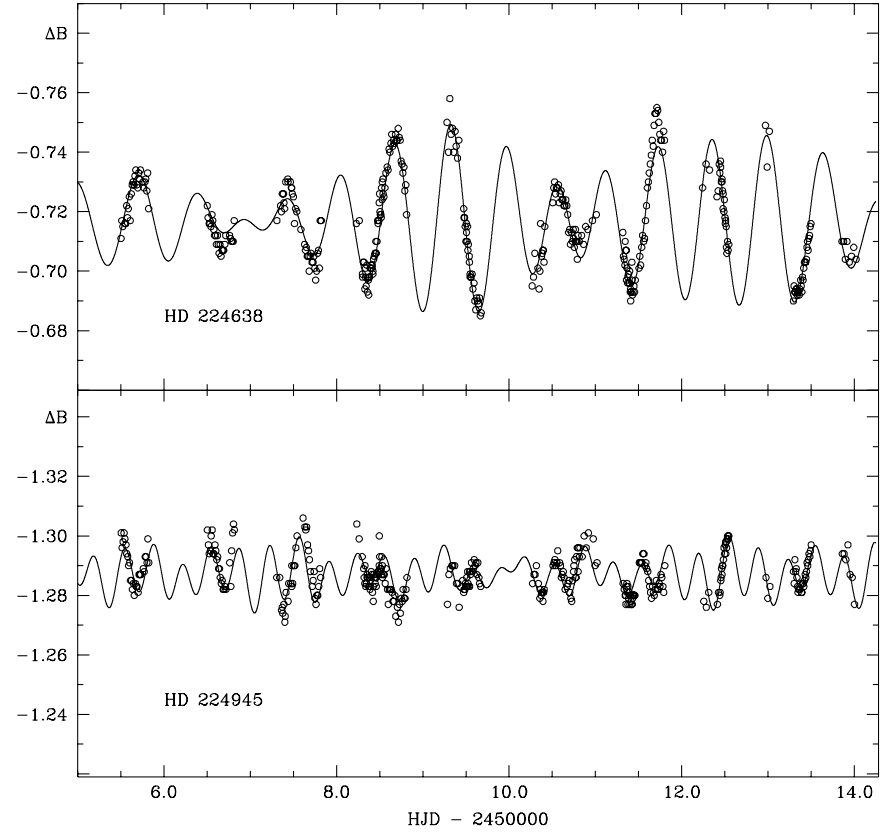

Fig. 1. Light curves ( $B$ light) of HD 224945 and HD 224638 obtained during the 1995 multisite campaign; measurements from JD 2450005 to 2450014 are shown. Note the different timescales and amplitudes between the two curves.

between HD 225086 and HD 200 cannot be used to correct magnitude differences between HD 225086 and the two program stars since they have different colours from HD 200; uncertainties of the colour transformations are larger than the precision we need. Hence, to apply this correction we exploited the very useful circumstance that the spread in longitude between different observing sites is not larger than the length of the night. There were always some overlapping segments of the light curve, which allowed us to determine the amounts of the corrections in a straightforward way.

It should also be noted that the Turkish measurements show a scatter larger than all the others. At first, all the Turkish data were considered, but in the last step of the analysis (once it was established that rapid variations are not present in the light curves) those measurements were averaged in groups of $4-5$. Thus, the final set of data contains the original measurements from all the observatories except for the averaged values from Turkey. The standard deviations of the $\Delta B$ and $\Delta V$ values between the comparison stars are 3.7 and 3.8 mmag, respectively.

Figure 1 shows a part of the $B$ light curves of HD 224945 and HD 224638. The fits derived in the next sections are also shown. The original measurements can be requested from the authors.

Since data in the final form circulated amongst all the participants, several co-authors analysed the time series independently and by different period search algorithms (least-squares, CLEAN, DFT, ...) and it was particularly satisfying to see that they detected the same terms, even if, owing to the complexity of the light variations, the terms did not always stand out in a clear way. 
Table 1. Summary of the photometric $B$ and $V$ data collected in the 1995 multisite campaign. $N$ is the total number of measurements, $n$ the number of nights. $t_{1}$ and $t_{N}$ are the times of the first and last measurement, respectively. Magnitude differences are calculated with respect to HD 225086.

\begin{tabular}{lrrrrrrrrrrr}
\hline \multicolumn{1}{c}{ Site } & \multicolumn{1}{c}{ Check star (HD 200) } & \multicolumn{3}{c}{ HD 224945 } & \multicolumn{4}{c}{ HD 224638 } \\
& $\Delta V$ & $N$ & s.d. & $t_{1}$ & $t_{N}$ & $n$ & $N$ & $t_{1}$ & $t_{N}$ & $n$ & $N$ \\
\hline ESO & 0.1472 & 198 & 0.0029 & 4.605 & 14.597 & 10 & 205 & 4.512 & 14.572 & 10 & 219 \\
Ege Obs. & 0.1180 & 117 & 0.0102 & 3.250 & 12.317 & 5 & 36 & 3.252 & 12.311 & 5 & 33 \\
SAAO & 0.1294 & 41 & 0.0055 & 1.427 & 14.479 & 6 & 36 & 1.432 & 14.482 & 6 & 44 \\
Mauna Kea & 0.1371 & 39 & 0.0051 & 7.824 & 14.003 & 6 & 37 & 7.840 & 14.023 & 6 & 38 \\
OSN & 0.1304 & 126 & 0.0032 & 7.364 & 19.524 & 9 & 128 & 7.362 & 19.527 & 9 & 133 \\
\hline & $\Delta B$ & $N$ & s.d. & $t_{1}$ & $t_{N}$ & $n$ & $N$ & $t_{1}$ & $t_{N}$ & $n$ & $N$ \\
\hline ESO & 0.2569 & 198 & 0.0029 & 4.604 & 14.597 & 10 & 203 & 4.512 & 14.572 & 10 & 217 \\
Ege Obs. & 0.2672 & 125 & 0.0091 & 3.264 & 12.312 & 5 & 32 & 3.251 & 12.322 & 5 & 29 \\
SAAO & 0.2458 & 41 & 0.0060 & 1.436 & 14.479 & 6 & 32 & 1.432 & 14.482 & 6 & 44 \\
Mauna Kea & 0.2554 & 20 & 0.0041 & 10.751 & 14.002 & 3 & 21 & 10.743 & 14.021 & 3 & 21 \\
OSN & 0.2449 & 126 & 0.0029 & 7.364 & 19.524 & 9 & 129 & 7.362 & 19.527 & 9 & 133 \\
\hline \hline
\end{tabular}

We present here the analysis carried out by using the least-squares method (Vaniĉek 1971) used by the Merate group in the analysis of $\delta$ Sct star light curves. This method has the advantage of not using any data prewhitening since only the frequency values previously found are considered as input values (known constituents; k.c.'s); their amplitude and phase values are recalculated as unknowns in the new searches. That means that after the detection of the $f_{1}$ term, only the frequency value $f_{1}$ was considered as established (i.e. a k.c.) and in the second search the unknowns were $\Delta m_{\mathrm{o}}, A_{1}, \phi_{1}, f_{2}, A_{2}, \phi_{2}$. The ordinates of the power spectra show the reduction factor

Red.Factor $=1-\frac{\sigma_{\text {fin }}^{2}}{\sigma_{\text {in }}^{2}}$.

Moreover, we can present the term detection step-bystep. The frequency values were refined after each new detection. We can also obtain useful evidence concerning the amplitude by applying a least-squares fit to the datasets. The interpolating formula we used is

$\Delta m(t)=\Delta m_{\mathrm{o}}+\sum_{i}^{N} A_{i} \cos \left[2 \pi f_{i}\left(t-T_{\mathrm{o}}\right)+\phi_{i}\right]$

Since the 1995 observations are concentrated in an interval of $15 \mathrm{~d}$ only, the frequency resolution is $1.5 / \Delta T=$ $0.10 \mathrm{~cd}^{-1}$. However, the absence of relevant aliases makes the detection of the excited terms very simple. We note that the frequencies mentioned in the text and figures are given to better accuracies that the formal frequency resolution, because they were determined by a least-squares procedure. The formal least-squares standard errors on the frequencies no doubt understimate the true uncertainties - aside from problems pointed out by e.g. Montgomery \& O'Donoghue (1999), it is clear that we have not always resolved close frequencies, and that some unidentified signals may remain in the data.

\section{Frequency analysis of the HD 224945 data}

\subsection{The 1995 campaign}

We collected $442 \quad V$ and $417 \quad B$ measurements of HD 224945, considering the Turkish measurements as binned points. The results of the frequency analysis of the $B$ data are shown in Fig. 2. In each panel the horizontal line indicates the level for $S / N=4.0$, i.e., the limit usually accepted for significance (Kuschnig et al. 1997).

The top panel shows the spectrum obtained without any k.c.: the peak at $3.00 \mathrm{~cd}^{-1}$ stands out clearly, and the alias structure mimics very well the spectral window (see also Fig. 1 in Poretti et al. 1996). In the discussion of the 1991 data (Paper I), the alias at $2.00 \mathrm{~cd}^{-1}$ was erroneously preferred. The reality of this term close to a multiple of $1 \mathrm{~cd}^{-1}$ was discussed in Paper I. We note that it is also detected in the new campaign data for HD 224945, but in neither the HD 224638, nor the HD 200, time series; its physical presence in the HD 224945 data is therefore certain. When introducing the $3.00 \mathrm{~cd}^{-1}$ frequency as k.c., the power spectrum shows an almost flat pattern, indicating that the amplitude of the remaining terms is smaller. However, two peaks reach the acceptance level: the first is located at $1.16 \mathrm{~cd}^{-1}$ (second panel), the second at $2.84 \mathrm{~cd}^{-1}$ (third panel). After introducing these three terms as k.c.'s, the detection of further terms becomes delicate. The highest peak in the fourth panel is at $2.42 \mathrm{~cd}^{-1}$ and after that the power spectrum does not show a really dominant peak (bottom panel).

The analysis of the $V$ measurements yields the same results, except with a slight difference in the frequency of the $2.84 \mathrm{~cd}^{-1}$ term. A value close to $2.77 \mathrm{~cd}^{-1}$ is preferred. Since the peak at $2.84 \mathrm{~cd}^{-1}$ is broad (see third panel of Fig. 2), the presence of another undetectable term combined with the noise distribution could be responsible for the difference between the two values. In the least-squares 

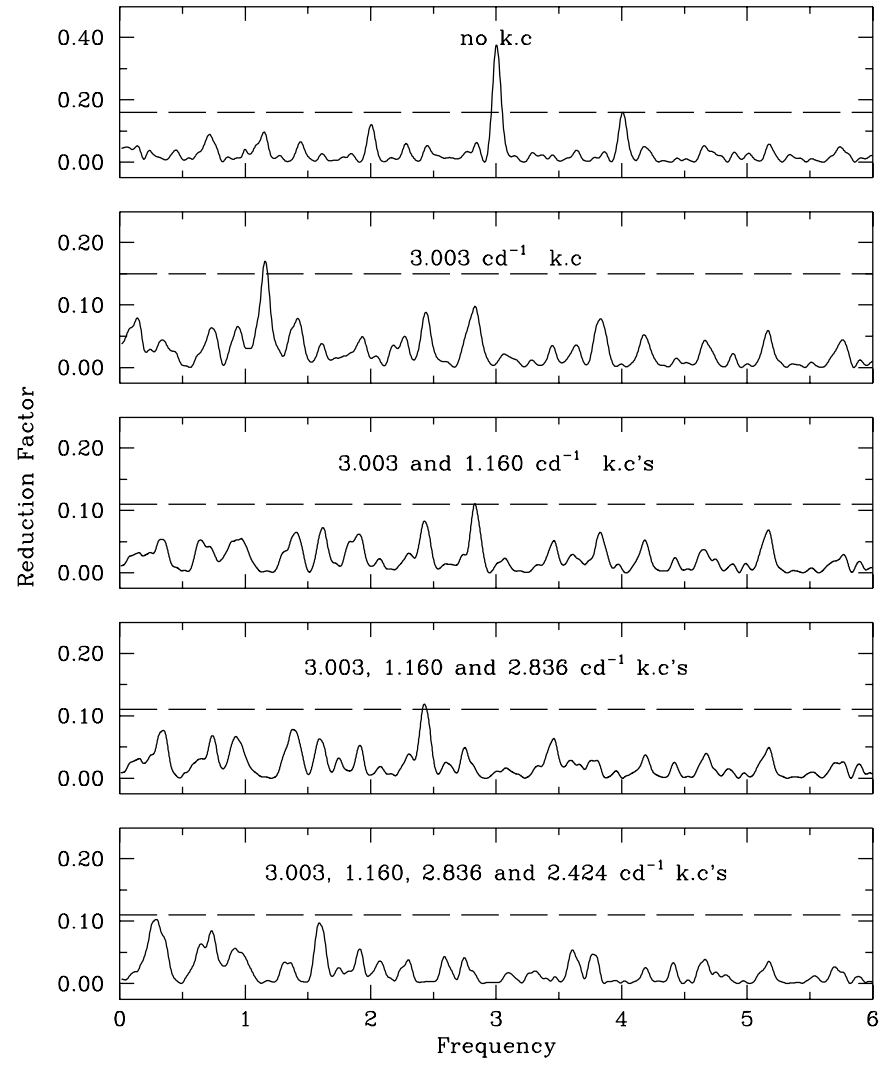

Fig. 2. Power spectra of the $B$ measurements of HD 224945 obtained during the 1995 multisite campaign. Note the different scale of the top panel.

solution we will consider the $2.84 \mathrm{~cd}^{-1}$ term as the only well-established term.

These independent terms describe a different scenario from that in Paper I. In that case the single-site observations make the power spectrum very complicated, with a great uncertainty between a peak and its $\pm 1 \mathrm{~cd}^{-1}$ aliases. This uncertainty led us to propose a solution based on two periodicities each having a triple-wave shape, as it was considered the simplest. Thanks to the multisite observations, we now know that many pulsational modes are simultaneously excited.

\subsection{The 1994 and 1991 observations}

The frequency analysis of the $B$ data collected in the 1994 season is much more complicated than that of the multisite campaign. In spite of that, we could detect the 3.00 and the $2.84 \mathrm{~cd}^{-1}$ terms. When introducing these two terms as k.c.'s, the highest peak is at $2.27 \mathrm{~cd}^{-1}$. This peak is significantly different than the $2.42 \mathrm{~cd}^{-1}$ detected in the 1995 multisite campaign. The search for a fourth component is not easy since we get numerous peaks: however, we could recognize the $1.16 \mathrm{~cd}^{-1}$ term and its aliases. We considered it as a real mode on the basis of the results of the 1995 campaign. After that, the new search showed evidence of a complicated structure centered at $1.66 \mathrm{~cd}^{-1}$. We have no way of selecting in a reliable way the true term amongst this peak and the aliased ones. Moreover, the $S / N$ of this term is less than 3.0. The $V$ data allowed us to identify the same terms, supporting the presence of the new term at $2.27 \mathrm{~cd}^{-1}$.

In the 1991 dataset the detection order of the terms is $3.00,2.84$ and $2.31 \mathrm{~cd}^{-1}$ : the only difference with respect to the previous least-squares search (see the beginning of Sect. 3.2 in Paper I) is the identification of the first term as $3.00 \mathrm{~cd}^{-1}$ instead of $2.00 \mathrm{~cd}^{-1}$. No other term can be detected after considering this triplet as k.c.'s; in particular there is no evidence of the presence of the $1.16 \mathrm{~cd}^{-1}$ term.

It should be emphasized that it would be very difficult to solve the 1994 and 1991 light curves without having the way shown by the results of the 1995 campaign. In turn, the similar structures observed in the three independent datasets corroborate the proposed identification of the excited frequencies, even if some of them are at the limit of the acceptance level.

\subsection{Frequency refinement and least-squares fitting}

The much longer time interval covered by the 1991 data (the two runs span 14 and 10 days, respectively, and they are separated by a gap of 10 days) allowed us to use these data to refine the frequency values. First, we calculated the best solution using all the 1991 data. Then we calculated the amplitudes for each 1991 subset keeping fixed frequencies and phases.

Before applying these frequency values to other datasets, we verified the correspondence between some terms. We considered the $2.27 \mathrm{~cd}^{-1}$ term detected in the 1994 data as the same detected at $2.31 \mathrm{~cd}^{-1}$ in the 1991 campaign. However, we note that the least-squares solution yields a better fit when considering different values for different seasons. On the other hand, we cannot consider the $2.42 \mathrm{~cd}^{-1}$ term detected in 1995 as coincident with the $2.31 \mathrm{~cd}^{-1}$ term. We suggest that there are many terms excited (simultaneously or not) in the narrow range $2.2-2.5 \mathrm{~cd}^{-1}$ and their detection is not an easy task. We considered the following five terms as independent modes detected in the three campaigns: 3.003, 2.836, 2.313, 2.424 and $1.160 \mathrm{~cd}^{-1}$. We also note the almost $2: 1$ ratio between the 2.313 and $1.160 \mathrm{~cd}^{-1}$ terms: the similar amplitude does not support a monoperiodic contribution to the light curve as it should be very asymmetric. The resonance effect is more plausible, even if it should be noted that the $1.160 \mathrm{~cd}^{-1}$ term was not observed in the 1991 dataset.

Using the four frequencies above, we calculated the amplitudes from the 1994 and 1995 datasets. Table 2 summarizes the results. The formal errors of the amplitudes are about 0.4 mmag. Looking at the differences between the amplitudes of the two 1991 subsets we can see that they are marginally significant, being in the interval $0.7-$ $0.9 \mathrm{mmag}$. This very close similarity demonstrates that solutions obtained from short runs are self-consistent. The dense time coverage in each dataset ensures the reliability of the amplitude determination. Therefore, we can look at 
Table 2. HD 224945: least-squares fit of the $B$ and $V$ measurements performed in the 1991, 1994 and 1995 observing seasons. The term detected at $2.27 \mathrm{~cd}^{-1}$ in the 1994 season is considered the same as that detected at $2.31 \mathrm{~cd}^{-1}$ in the other seasons, while that at $2.42 \mathrm{~cd}^{-1}$ is considered an independent frequency. Formal errors on the frequencies are calculated with respect to the 1991 data (when applicable) or to the 1995 data.

\begin{tabular}{|c|c|c|c|c|c|c|c|}
\hline \multirow{3}{*}{$\begin{array}{l}\text { Freq. } \\
{\left[\mathrm{cd}^{-1}\right]}\end{array}$} & \multicolumn{5}{|c|}{ Amplitude $B$ [mmag] } & \multicolumn{2}{|c|}{ Amplitude $V$ [mmag] } \\
\hline & 1991 & 1991 & 1991 & 1994 & 1995 & 1994 & 1995 \\
\hline & All & I & II & & & & \\
\hline $3.00 \pm 0.001$ & 4.3 & 4.5 & 3.8 & 5.4 & 6.1 & 4.0 & 5.1 \\
\hline $2.836 \pm 0.001$ & 4.9 & 5.2 & 4.3 & 4.0 & 2.5 & 3.4 & 1.5 \\
\hline $2.313 \pm 0.001$ & 4.3 & 4.5 & 3.8 & 2.5 & - & 2.3 & - \\
\hline $2.424 \pm 0.005$ & - & - & - & - & 2.3 & - & 1.9 \\
\hline $1.160 \pm 0.005$ & - & - & - & 2.5 & 3.0 & 2.2 & 2.4 \\
\hline Res. rms [mmag] & 5.4 & 5.3 & 5.4 & 5.1 & 4.2 & 5.0 & 4.2 \\
\hline
\end{tabular}

the differences in the amplitudes between the 1991, 1994 and 1995 datasets with greater confidence.

We also analyzed in frequency the data obtained by merging the 1991, 1994 and 1995 datasets. For each detected term, it is not possible to select the true value owing to the numerous aliases separated by integer values of $\pm 1 \mathrm{cy}^{-1}$; therefore, in the case of HD 224945 we cannot propose a full, comprehensive solution and evaluate in a reliable way the phase coherence of the pulsation. The amplitude variability is a further complication. It should be noted that an attempt at such an analysis detected two peaks at 2.27 and $2.31 \mathrm{~cd}^{-1}$, suggesting that these two peaks are related to two independent terms (see the above comparison of the 1994 and 1991 results).

The sum of the squared amplitudes are the same in 1991 and 1994 (61 and $58 \mathrm{mmag}^{2}$, respectively), while it is lower in $1995\left(50 \mathrm{mmag}^{2}\right)$. However, the amplitude of a mode can change dramatically: the $2.836 \mathrm{~cd}^{-1}$ term halved its $B$ amplitude from 1991 to 1995 while the $2.313 \mathrm{~cd}^{-1}$ term disappeared. As a matter of fact, in the 1991 dataset there are three terms having the same amplitude, while in the 1995 ones the $3.003 \mathrm{~cd}^{-1}$ term is dominating. The amplitude variability looks like a wellestablished fact for this $\gamma$ Dor star.

\section{Frequency analysis of the HD 224638 data}

\subsection{The 1995 campaign}

The analysis of the most recent $B$ and $V$ measurements of HD 224638 identified the same terms as before, i.e., 1.627, $1.368,1.697,1.565$ and $1.145 \mathrm{~cd}^{-1}$ (Fig. 3). Also in this case the multisite observations allowed us to identify the terms without any ambiguity. Differently than the case of HD 224945, there are several very close frequencies: 1.627 (top panel), 1.697 (third panel), and $1.565 \mathrm{~cd}^{-1}$ (fourth panel). The separations between the two side peaks and the central one are 0.07 and $0.06 \mathrm{~cd}^{-1}$; these values are comparable to the half width at the half maximum, i.e. $1 / \Delta T=0.07 \mathrm{~cd}^{-1}$. Indeed, in the power spectra the peaks are not well resolved, but they appear to be double and/or enlarged. In particular, the double peaks appearing in the

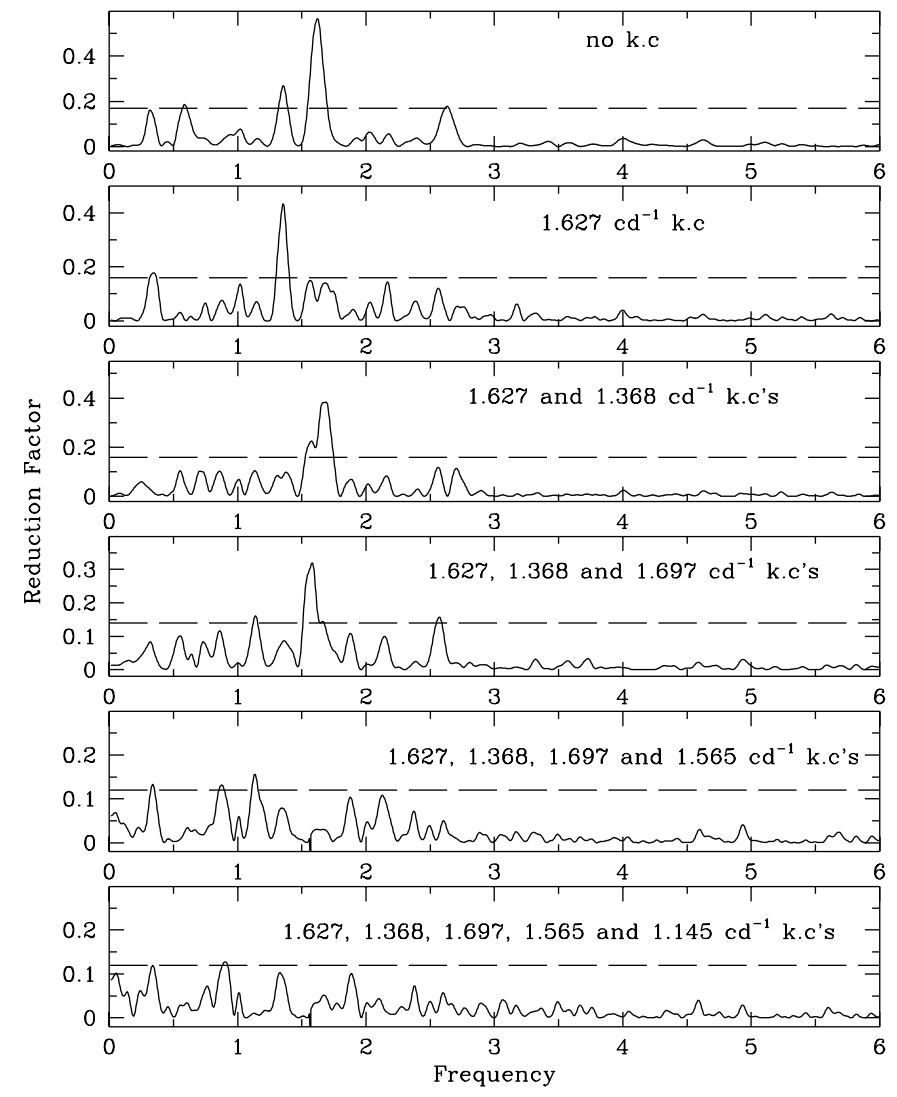

Fig. 3. Power spectra of the $B$ measurements of HD 224638 obtained during the 1995 multisite campaign. Note the different scales of the panels.

third and fourth panels of Fig. 3 should be noted. This reveals the presence of overlapping peaks. Two peaks flanking a central one at the limit of the frequency resolution can be generated by a single term modulated in amplitude; however, the amplitudes of the side peaks are comparable to that of the central one and this fact supports the reality of the triplet. The analysis of the 1991 dataset definitely confirms the hypothesis of the three independent modes (see next subsection). Note that the amplitudes of these terms are larger than those detected in the HD 224945 
Table 3. HD 224638: least-squares fit of the $B$ and $V$ measurements performed in the 1991, 1994 and 1995 observing seasons. Formal errors on frequencies are calculated with respect to the 1991 data.

\begin{tabular}{|c|c|c|c|c|c|c|c|}
\hline \multirow{3}{*}{$\begin{array}{l}\text { Freq. } \\
{\left[\mathrm{cd}^{-1}\right]}\end{array}$} & \multicolumn{5}{|c|}{ Amplitude $B$ [mmag] } & \multicolumn{2}{|c|}{ Amplitude $V$ [mmag] } \\
\hline & 1991 & 1991 & 1991 & 1994 & 1995 & 1994 & 1995 \\
\hline & All & $\bar{I}$ & II & & & & \\
\hline $1.627 \pm 0.001$ & 9.1 & 9.0 & 8.9 & 7.1 & 10.8 & 6.2 & 9.2 \\
\hline $1.368 \pm 0.001$ & 9.0 & 8.4 & 8.7 & 7.3 & 10.2 & 6.0 & 7.4 \\
\hline $1.697 \pm 0.001$ & 8.0 & 7.1 & 9.4 & 7.7 & 7.4 & 6.6 & 6.3 \\
\hline $1.565 \pm 0.001$ & 4.7 & 5.0 & 6.0 & 9.1 & 5.2 & 7.0 & 4.0 \\
\hline $1.145 \pm 0.001$ & 2.1 & 2.6 & 4.5 & 2.1 & 3.0 & 1.7 & 2.1 \\
\hline Res. rms [mmag] & 6.4 & 4.9 & 7.1 & 5.6 & 4.7 & 5.1 & 4.3 \\
\hline
\end{tabular}

data, giving a higher $S / N$ value. The other two terms (1.368, second panel, and $1.145 \mathrm{~cd}^{-1}$, fifth panel) are more separated.

The power spectrum obtained by introducing the five frequencies as k.c.'s is not homogenously flat (bottom panel). The noise in the region $0.0-2.0 \mathrm{~cd}^{-1}$ is $1.34 \mathrm{mmag}$, while in the region $4.0-6.0 \mathrm{~cd}^{-1}$ it is only $0.65 \mathrm{mmag}$. However, it should be noted that the general patterns of the residual $B$ and $V$ power spectra are slightly different. This fact suggests that noise dominates over pulsation and the latter is really undetectable owing to the very small amplitude of the involved terms.

\subsection{The 1994 and 1991 seasons}

Once again, the light curves of the 1991 and 1994 seasons can only be understood if extra alias-free frequency information is available. The frequency analysis of the $1991 B$ data provided evidence for the terms 1.697, 1.627, 1.368 and $1.565 \mathrm{~cd}^{-1}$. The first three terms were already reported in Paper I, where two periodicities having each the shape of a double--wave were proposed. The $1.565 \mathrm{~cd}^{-1}$ (detected also in the 1995 dataset) is a new term obtained by pushing one further step in the analysis. As a result, we also detect here the close triplet observed in the 1995 campaign. Since the frequency resolution in the 1991 dataset is much better than in the 1995 one, this confirmation strengthens our confidence in the reality of the triplet. The peak at $1.145 \mathrm{~cd}^{-1}$ does not appear very clearly in the power spectrum, but it has been considered for the least-squares solution.

The first four terms are also detected in the $1994 B$ and $V$ datasets, but residual signal is left, especially in the region $0.5-1.0 \mathrm{~cd}^{-1}$. The $1.145 \mathrm{~cd}^{-1}$ term shows an amplitude much smaller than the other four terms. As a matter of fact, in this season it seems that many terms having amplitudes at the mmag level are excited.

\subsection{Frequency refinement and least-squares fitting}

We followed the same procedure used for HD 224945, refining the frequency values by means of the 1991 dataset and then calculating the amplitudes for the two 1991 subsets and the other datasets. The frequency analysis already gave some hint about the amplitude variability of some terms, since for example the $1.145 \mathrm{~cd}^{-1}$ peak was not clearly detected in all datasets.

This is confirmed by the large amplitude variability, which can be discerned from Table 3. Considering the 1991 subsets we can see differences of up to $2.3 \mathrm{mmag}$, but note the $+3.7,+2.9$ and -3.9 mmag differences between the amplitudes of the $1.627,1.368$ and $1.565 \mathrm{~cd}^{-1}$ terms in 1995 and 1994 datasets, which look extremely large. The term ranking is also largely changing. Note that the largest amplitude of the $1.565 \mathrm{~cd}^{-1}$ term was in 1994 , whereas usually this term was below the first three in 1991 and 1995. The amplitude is also greatly changing. The sum of the squared amplitudes is higher in the 1995 data $\left(311 \mathrm{mmag}^{2}\right)$ and lower in the 1994 dataset $\left(250 \mathrm{mmag}^{2}\right)$, with a variation of $25 \%$. This difference supports the intrinsic variability of the mode amplitudes. Note for example that in the 1991 data there are three terms having a large amplitude, in 1994 data there are four and in 1995 data two of them clearly have the largest amplitude, considerably greater than the others. Hence, the amplitude changes look more conspicuous in HD 224638 than in HD 224945.

In the case of HD 224638 we can merge the 1991, 1994 and 1995 datasets knowing that all the datasets show the same frequency content. Indeed, the frequency analysis of the whole set yields the same frequencies listed in Table 3. The detection of the three terms at 1.565, 1.627 and $1.697 \mathrm{~cd}^{-1}$ supports the identification as three independent modes proposed in Sect. 5.1. Unfortunately, we cannot give refined values for the frequencies, owing to the $\mathrm{cy}^{-1}$ aliases. However, we performed some tests assuming a constant value for the frequency and then calculated the amplitudes and phases of each term for each observing season. Besides the verification of the amplitude variability, we found that the same frequency displays similar phase values from one season to the next. This fact supports an intrinsic variability of the mode amplitudes, rather than a beating phenomenon between two very close terms. 


\section{Physical properties}

The properties of $\gamma$ Dor stars have been reviewed by Zerbi (2000); driving mechanisms have been proposed by Guzik et al. (2000), Wu (2002) and Löffler (2002), but the origin of the pulsation in $\gamma$ Dor stars still remains an open problem.

HD 224945 and HD 224638 have very similar physical properties (Zerbi 2000): $M_{V}=2.98, L / L_{\odot}=5.5, T_{\text {eff }}=$ $7200 \mathrm{~K}, R / R_{\odot}=1.51$ and $M / M_{\odot}=1.52$ for HD 224638, $M_{V}=3.07, L / L_{\odot}=5.1, T_{\text {eff }}=7250 \mathrm{~K}, R / R_{\odot}=1.43$ and $M / M_{\odot}=1.51$ for HD 224945. Also the difference in metallicity is not significant, $[\mathrm{Me} / \mathrm{H}]=-0.15$ vs. -0.30 , respectively. The two stars occupy very similar positions also in the colour-magnitude diagram, being located on the ZAMS, in the middle of the domain of $\gamma$ Dor stars and on the low temperature edge of the $\delta$ Sct region (Handler 1999). Therefore, two very similar stars display different pulsational modes, since HD 224945 is characterized by four frequencies spread over a large interval, while HD 224638 displays a much more closely spaced set (it is similar to HR 2740; see Poretti et al. 1997). Moreover, the residual power spectrum of HD 224638 has a very low noise level above $4.0 \mathrm{~cd}^{-1}$, while that of HD 224945 has a higher level, suggesting some signal contribution.

The large spread in the frequency values observed for HD 224945 is another piece of evidence that the cause of light variability in these stars is pulsation and not stellar activity. Differential rotation is not able to match a spread in frequencies as large as $0.7 \mathrm{~cd}^{-1}$ (considering the three terms always observed, i.e., not considering the even more distant frequency at $\left.1.16 \mathrm{~cd}^{-1}\right)$.

We note that multiperiodicity is not observed in all $\gamma$ Dor stars. Some display only a single photometric period (i.e., a single low-order mode). A relevant example is HD $207223 \equiv \operatorname{HR} 8330$ (Aerts \& Kaye 2001), which is also monoperiodic from a spectroscopic point of view (i.e. it does not show any high-order modes). Another example is HD 164615 (Zerbi et al. 1997), one of the first known $\gamma$ Dor stars. It is probably a monoperiodic variable which shows amplitude modulation.

Finally, we note that HD 224945 exhibits the shortest known period (i.e. $0.3330 \mathrm{~d}$ ) of the $\gamma$ Dor stars. Guzik et al. (2000) considered $0.48 \mathrm{~d}$ as the lower limit (probably the old value of the same term, i.e. $2.00 \mathrm{~cd}^{-1}$, Paper I). Therefore, the period value cannot be used to separate $p$ - and $g$-modes, as $\delta$ Sct stars can display such "long-period" $p$-modes. A careful evaluation of the physical parameters is necessary. Indeed, in HD 224638 and HD 224945 the fundamental radial mode is shorter than $0.07 \mathrm{~d}$, confirming that $p$ - and $g$-modes are well separated in $\gamma$ Dor stars.

\section{Conclusions}

The solution of the light curves of HD 224638 and HD 224945 has been deduced only on the basis of a multisite campaign, since the true peaks could not be recognized if $\mathrm{a} \pm 1 \mathrm{~cd}^{-1}$ effect is present in the spectral window. We did our best to identify the correct excited modes, but the more important characteristic of these two stars is the strong multiperiodicity itself, independent of the exact frequency values. In our opinion, HD 224638 and HD 224945 provide two of the best examples of features typical of multiperiodicity among $\gamma$ Dor variables: different sets of frequency content, amplitude variations, disappearing terms, close doublets of frequencies. Also, after detecting five or six terms, the rms scatter is larger than the observational error. Therefore, residual signal is hidden in the noise. Such extreme multiperiodicity can be clearly ascribed to pulsation, as stellar activity is not able to generate it.

The differences in the frequency and amplitude ranges of HD 224638 and HD 224945 (clearly visible in Fig. 1) remind us of the unpredictable frequency content of $\delta$ Sct stars, where the selection mechanism among all the possible modes seems to be different from one star to the next (Poretti 2000). The amplitude variability of the excited modes is another point of similarity between $\gamma$ Dor and $\delta$ Sct stars. Other than multiperiodicity, HD 224945 and HD 224638 provide the best examples of amplitude variability among $\gamma$ Dor stars, following the three campaigns carried out on these stars. This observational evidence suggests strategies for asteroseismological space missions: a long observing run (or two separate runs) may be very helpful in detecting more terms (excited at different levels at different times) or in studying damping effects.

Acknowledgements. ER and SM acknowledge the partial support by the Junta de Andalucia and by the Direccion General de Investigacion (DGI) under project AYA2000-1559. SM also acknowledges the financial support by the Osservatorio Astronomico di Brera and by the Agenzia Spaziale Italiana (ASI Contract I/R/037/01). Thanks are due to an anonymous referee and to M. Breger for useful comments and suggestions on the first version of the manuscript.

\section{References}

Aerts, C., \& Kaye, A. B. 2001, ApJ, 553, 814

Balona, L. A., Krisciunas, K., \& Cousins, A. W. J. 1994, MNRAS, 270, 914

Balona, L. A., Böhm, T., Foing, B. H., et al. 1996, MNRAS, 281,1315

Guzik, J. A., Kaye, A. B., Bradley, P. A., Cox, A. N., \& Neuforge, C. 2000, ApJ, 542, L57

Handler, G. 1999, MNRAS, 309, L19

Kaye, A. B., Handler, G., Krisciunas, K., Poretti, E., \& Zerbi, F. M. 1999, PASP, 111, 840

Krisciunas, K., Griffin, R. F., Guinan, E. F., Luedeke, K. D., \& McCook, G. P. 1995, MNRAS, 273, 662

Kuschnig, R., Weiss, W. W., Gruber, R., Bely, P. Y., \& Jenkner, H. 1997, A\&A, 328, 544

Löffler, W. 2002, in ASP Conf. Ser., Radial and Nonradial Pulsations as Probes of Stellar Physics, ed. C. Aerts, T. R. Bedding, \& J. Christensen-Dalsgaard, in press 
Mantegazza, L., \& Poretti, E. 1991, IBVS, 3690

Mantegazza, L., Poretti, E., \& Bossi, M. 1996, A\&A, 308, 847

Mantegazza, L., Poretti, E., \& Zerbi, F. M. 1994, MNRAS, 270, 439, Paper I

Montgomery, M., \& O’Donoghue, D. 1999, Delta Scuti Star Newsletter, 13, 26

Poretti, E. 2000, in Delta Scuti and Related Stars, ed. M. Breger, \& M. H. Montgomery, ASP Conf. Ser., 210, 45

Poretti, E., Akan, C., Bossi, M., Koen, C., Krisciunas, K., \& Rodríguez, E. 1996, in Sounding Solar and Stellar Interiors, IAU Symp. 181, Poster Volume, ed. J. Provost, \& F. X. Schmider, 279
Poretti, E., Koen, C., Martinez, P., Breuer, F., de Alwis, D., \& Haupt, H. 1997, MNRAS, 292, 621

Poretti, E., \& Zerbi, F. M. 1993, A\&A, 268, 369

Vaniĉek, P. 1971, Ap\&SS, 12, 10

Wu, Y. 2002, in ASP Conf. Ser., Radial and Nonradial Pulsations as Probes of Stellar Physics, ed. C. Aerts, T. R. Bedding, \& J. Christensen-Dalsgaard, in press

Zerbi, F. M., Rodríguez, E., Garrido, R., et al. 1997, MNRAS, 292,43

Zerbi, F. M. 2000, in Delta Scuti and Related Stars, ed. M. Breger, \& M. H. Montgomery, ASP Conf. Ser., 210, 332 